\title{
High Speed Wireless Video Transmission for Handheld Ultrasonic System
}

\author{
Jiang Dai \\ Shenzhen institutes of advanced \\ technology, Chinese academy of \\ science, Shenzhen, China \\ Inner Mongolia University, Hohhot, \\ China \\ 2871513143@qq.com
}

\author{
Xuemei Lei \\ Inner Mongolia University, Hohhot, \\ China \\ ndlxm@imu.edu.cn
}

\author{
Fengqi $\mathrm{Yu}^{*}$ \\ Shenzhen institutes of advanced \\ technology, Chinese academy of \\ science, Shenzhen, China \\ fq.yu@siat.ac.cn
}

\begin{abstract}
Portable ultrasound equipment has been widely used in emergency, bedside patrol, and chronic disease management. Higher requirements have been put forward for some applications. However, the existing portable ultrasonic equipment still has some problems, such as low wireless transmission rate, high power consumption, and unreliable wireless transmission. To resolve these problems, we propose and implement a wireless transmission system for handheld ultrasonic application. With embedded Linux as the operating system, the paper describes the circuit design and its implementation, as well as system software design. The designed system has been tested. The experiment results show that the transmission rate of the system can reach $367 \mathrm{Mbps}$. In addition, the system has low power consumption. The stable transmission distance can reach up to $9 \mathrm{~m}$.
\end{abstract}

\section{CCS CONCEPTS}

- Information systems; • Information systems applications; - Multimedia information systems; • Multimedia streaming;

\section{KEYWORDS}

Portable ultrasound, WiFi, Wireless transmission

\section{ACM Reference Format:}

Jiang Dai, Xuemei Lei, and Fengqi Yu*. 2021. High Speed Wireless Video Transmission for Handheld Ultrasonic System. In The Fifth International Conference on Biological Information and Biomedical Engineering (BIBE2021), fuly 20-22, 2021, Hangzhou, China. ACM, New York, NY, USA, 4 pages. https://doi.org/10.1145/3469678.3469709

\section{INTRODUCTION}

With the rapid development of embedded and wireless communication technology, the combination of the two brings many new applications. One of the them is in the medical field. For traditional B-ultrasound equipment, which is bulky and has poor mobility, patients need to make an appointment for ultrasound examination. In

Permission to make digital or hard copies of all or part of this work for personal or classroom use is granted without fee provided that copies are not made or distributed for profit or commercial advantage and that copies bear this notice and the full citation on the first page. Copyrights for components of this work owned by others than ACM must be honored. Abstracting with credit is permitted. To copy otherwise, or republish, to post on servers or to redistribute to lists, requires prior specific permission and/or a fee. Request permissions from permissions@acm.org.

BIBE2021, July 20-22, 2021, Hangzhou, China

(c) 2021 Association for Computing Machinery.

ACM ISBN 978-1-4503-8929-7/21/07 . \$15.00

https://doi.org/10.1145/3469678.3469709 addition, the transmission of ultrasonic video is also by the means of cables which largely limits the flexibility of B-ultrasound equipment and thus fails to meet the requirement of doing examination at any time. This is especially difficult for patients who are unable to move.

The future medical devices will become smaller, easy to carry, and doing checkup at any time. There are several portable ultrasound devices using wireless transmission in the market. In 2009, Siemens introduced the Acuson P10 portable ultrasound device, which is small, light, and with display size of a mobile phone. Its emergence represents the era of the portable ultrasonic equipment [1]. In 2011, General Electric company launched Vscan with color ultrasound imaging compared with white and black display of Acuson P10 [2]. Using USB3.0 interface, literature [3] proposed method to transfer the processed ultrasound images to a tablet computer which performs back-end processing and image display. The above-mentioned three kinds of ultrasonic equipment all transmit B-ultrasonic images by means of cable transmission, which brings inconvenience in some applications. In literature [4], smart phones were proposed to process B-ultrasound images, such as orthogonal demodulation and scanning conversion. It can further reduce the data amount of B-ultrasonic equipment. In 2013, Siemens took the lead in launching Acuson Freestyle, which uses 7.8GHz UWB wireless transmission. The transmission distance was 3 meters [5]. In 2016, domestic Sonostar company launched UProbe and Xprobe wireless ultrasonic devices, which use $2.4 \mathrm{GHz}$ WiFi to transmit images in real time [6].

The portable ultrasound device is usually battery-powered, so it has strict requirements of high transmission rate, low power consumption, and high reliability. The motivation of the paper is to resolve the above problems simultaneous.

The rest of the paper is organized as follows. The design of the system hardware and software are presented in section II and III respectively. Section IV shows the system test. Section V is the conclusion.

\section{DESIGN OF SYSTEM HARDWARE}

\subsection{Hardware Structure Design}

There are mainly two kinds of interfaces available for high-rate wireless communication module: USB interface and SDIO interface. The ultrasonic video has higher requirements for communication stability due to the connection failure in real-time video transmission. Compared with the USB interface, the SDIO interface has good 
stability under the same environment. Therefore SDIO interface is adopted in our design. The video resolution of today's B-ultrasonic equipment is gradually improving, so the transmission bandwidth needs to be improved as well to meet the requirement of video transmission rate. On the other hand, low power consumption is required for extending the service time of the battery.

The MCU and RF module is carefully considered in the system design. After comparison, the AP6356S wireless module based on Broadcom BCM4356 is adopted. This module supports 802.11ac protocol and can work at $5 \mathrm{GHz}$. The theoretical transmission rate of SDIO can reach $866 \mathrm{Mbps}$ which can meet our requirements. The simplified block diagram of the transmitter is shown in Figure 1

\subsection{Hardware Circuit Design}

In our circuit design, the AP6356S wireless communication module is connected to RK3399 microcontroller through SDIO interface. It communicates with RK3399 controller through SDIO. The SDIO_DATA_0,SDIO_DATA_1,SDIO_DATA_2 and SDIO_DATA_3 of the data transmission pins of AP6356S module are connected with GPIO2_C4, GPIO2_C5, GPIO2_C6 and GPIO2_C7 of the RK3399, respectively. The clock pin SDIO_DATA_CLK is connected to GPIO2_D0 and the command pin SDIO_DATA_CMD is connected to GPIO2_D1 of RK3399 respectively. The XTAL_IN/OUT pin requires an external $37.4 \mathrm{MHz}$ crystal oscillator to generate clock signal. WIFI_HOST_WAKE_L is connected to GPIO0_A3 and WIFI_REG_ON_H is connected to GPIO0_B2 of RK3399 respectively. The Bluetooth function is not activated in this design.

The power voltage used in this design is as follows. The power supply voltage of RK3399 chip is $5.0 \mathrm{~V}$. The wireless module needs $3.3 \mathrm{~V}$ and VDDIO needs $1.8 \mathrm{~V}$. MP2143 chip is used to generate $3.3 \mathrm{~V}$ A $1 \mathrm{uH}$ inductor and two parallel $22 \mathrm{uF}$ capacitors are used to filter out the noise of the power supply.

\subsection{Antenna Design}

This design adopts FR05-S1-NO-1-003 antenna of Fractus. This antenna has the characteristics of low cost, dual-band support, small size, and easy to be integrated into PCB board. We use two antennas to support MIMO with the selected wireless module, so as to maximize its transmission rate capability. In addition it supports spatial diversity and can effectively improve the reception quality of signals. According to AP6356S and antenna requirements, we need to achieve impedance matching of $50 \mathrm{ohms}$ at $5 \mathrm{GHz}$ frequency. The overall circuit is shown in Figure 2

\section{DESIGN OF SYSTEM SOFTWARE}

\subsection{System software design}

Linux system has the characteristics of completely open source, strong system portability, good real-time performance, convenient to be tailoring, and strong compatibility for different control chips. Therefore, the software of this design is running in the Linux environment.

The software mainly includes the design of the lower-layer software, including U-Boot porting, the porting of Linux embedded system, Rootfs building, the driver modification of wireless module, etc., to completely match with the hardware platform. The whole process is described as follows [7].

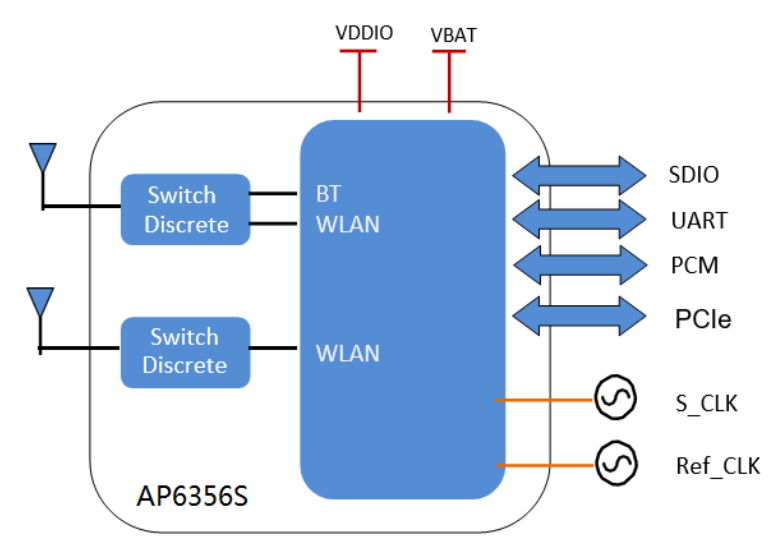

Figure 1: Simplified Block Diagram of the Transmitter.

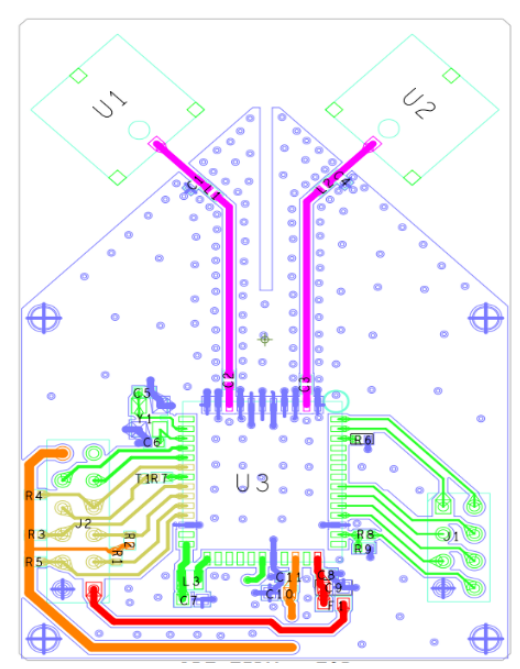

Figure 2: Overall Circuit Design.

Copy the Uboot source code provided by the development board to the virtual machine. Select the deconfig file corresponding to the RK3399 development board to configure the system. Execute the make command, compile the Uboot, and get its image file.

Extract the kernel source code to the directory specified by the virtual machine. Execute the make menuconfig command to select the configuration, and execute the make command to generate the kernel image file for the development board.

Enter the Buildroot file. Execute the make menuconfig command to configure the root file system. Execute make command to compile and generate the required root file system.

Modify the driver source code of the wireless module. In the makefile, replace LINUX_SRC with the path where the development board kernel source code is located. CROSS_COMPILE is replaced with a cross-compiled path. After the modification is complete, execute the make command to generate the driver file. 


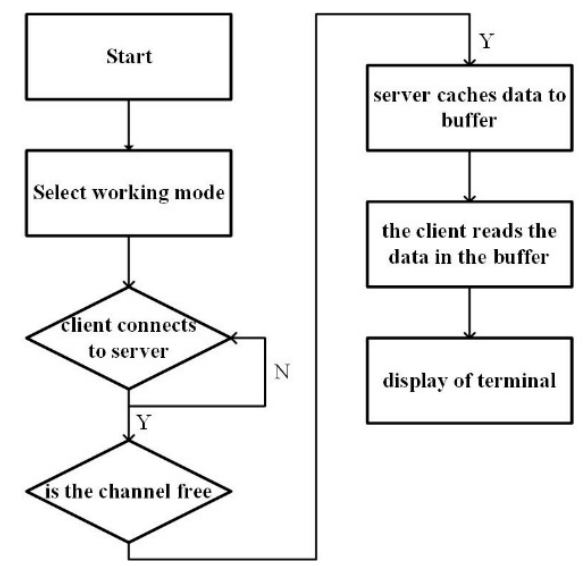

Figure 3: Flowchart of the Application Software.

\subsection{System Working Mode and Transport Protocol}

The wireless handheld ultrasound system can work in two modes: station and AP (Access Point) modes. The station mode is defined as that the handheld ultrasound detector is working in station mode and mobile display terminal is connected to an AP. The ultrasonic image is transmitted to the mobile display terminals in real time through the AP. The AP mode is defined as that the handheld ultrasound detector is configured as AP mode and the mobile display terminals are wirelessly connected to it. The B-ultrasound image can be sent to the display terminals from the handheld ultrasound detector directly. The transport protocol can adopt UDP (User Datagram Protocol) and TCP (Transmission Control Protocol). UDP is an unreliable connection mode. Packet loss may occur at the receiving end. But the transmission rate is faster and the real-time performance is better. In the TCP mode, the receiver is required to return an acknowledgement packet after receiving the IP packet. If the sender does not receive the acknowledgement packet, it has the retransmission mechanism. TCP has a lower transmission rate, a certain delay, and occupies more system resources [8]. In our system, both TCP and UCP mode are studied and tested.

\subsection{Application Software Design}

The flowchart of the application software is shown in Figure 3 This software design can configure the system to AP or station mode. When the system is configured as AP mode, it can support the connection of multiple mobile display terminals to facilitate the communication and consultation among doctors. In the flowchart, first, the working mode is selected. Then the connection between the mobile terminal and the wireless module is checked. In the AP mode, multiple mobile terminals are wirelessly connected to the ultrasonic equipment, i.e. AP. The AP needs to detect whether the wireless channel is idle. When the channel is detected to be idle, the ultrasonic device caches the data into the buffer and sends out. The mobile terminal reads the data and displays the ultrasonic video on the screen in real time. In the station mode, the handheld ultrasound detector is wirelessly connected to an AP which is connected to a display screen. The handheld ultrasound device detects network

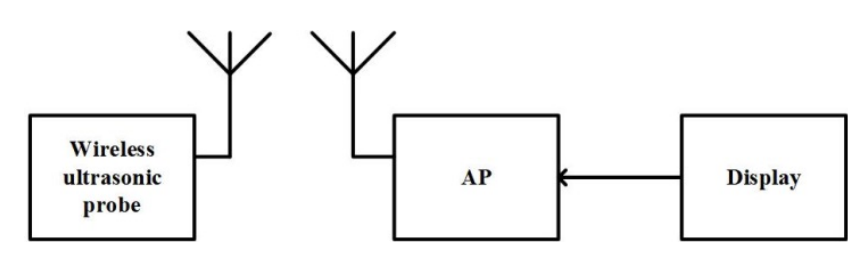

Figure 4: Test Setup for Station Mode.

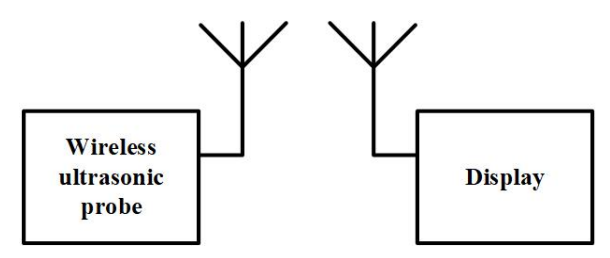

Figure 5: Test Setup for AP Mode.

channel condition. If the channel quality is poor, the data waits until the channel becomes better. Then the data is sent out to the AP in real time.

\section{SYSTEM TESTING}

The test of the system is conducted in two ways: station mode and AP mode for UDP and TCP respectively.

\subsection{Test Setup}

The test setup for station mode is shown in Figure 4. Portable ultrasound detector communicates with the AP wirelessly. The display device is wired to the AP. The AP automatically assign IP addresses to the display device.

The test setup for AP mode is shown in Figure 5. The handheld ultrasound terminal is first configured to AP mode, and the PC or other mobile terminals are connected to the AP in a wireless manner, without the need for the hospital to deploy the network environment.

\subsection{Speed Test}

The iperf, which is a software tool for network performance measurement, is used for measuring the data rate. The network speed measurement is conducted for both UDP and TCP protocols.

The data rate for station mode and UDP protocol: the DUT (device under test) is set to UDP. The iperf can automatically test the data bandwidth between the client and the server. In the meantime, it measures the corresponding packet loss. The tested transmission rate can go up to $367 \mathrm{Mbps}$. The data rate for station mode and TCP protocol: the DUT is set to TCP. The tested transmission rate can go up $304 \mathrm{Mbps}$.

The data rate for AP mode and UDP protocol: the DUT is set to UDP. The tested transmission rate can go up $348 \mathrm{Mbps}$. The data rate for AP mode and TCP protocol: the DUT is set to TCP. The tested transmission rate can go up $298 \mathrm{Mbps}$.

There is a data rate difference between UDP and TCP. This is because TCP is a reliable transmission, which adopts the connection 
Table 1: Test Result of Distance, Packet Loss, and Data Rate for Station Mode and UDP Protocol.

\begin{tabular}{llll}
\hline Distance & $100 \mathrm{Mbps}$ & $200 \mathrm{Mbps}$ & $300 \mathrm{Mbps}$ \\
\hline $\mathbf{3 m}$ & $0.75 \%$ & $1.60 \%$ & $1.70 \%$ \\
$\mathbf{6 m}$ & $1.80 \%$ & $2.30 \%$ & $2.20 \%$ \\
$\mathbf{9 m}$ & $2.20 \%$ & $2.20 \%$ & $3.64 \%$ \\
\hline
\end{tabular}

Table 2: Test Result of Distance, Packet Loss, and Data Rate for AP and UDP Protocol.

\begin{tabular}{llll}
\hline Distance & $100 \mathrm{Mbps}$ & $200 \mathrm{Mbps}$ & $300 \mathrm{Mbps}$ \\
\hline $\mathbf{3 m}$ & $0.08 \%$ & $0.10 \%$ & $0.64 \%$ \\
$\mathbf{6 m}$ & $0.83 \%$ & $0.75 \%$ & $1.27 \%$ \\
$\mathbf{9 m}$ & $0.69 \%$ & $0.79 \%$ & $2.63 \%$ \\
\hline
\end{tabular}

of handshaking. On the other hand, UDP is an unreliable transmission, where data is transmitted without acknowledgment. So UDP has higher transmission data rate.

\subsection{Transmission Distance Test}

Transmission distance is an important parameter in our system. The test results for station mode and UDP protocol are shown in Table 1.

The test results for AP configuration and UDP mode are shown in Table 2 .

It can be seen from the tables that compared with the 3-meter transmission distance of Siemens Acuson Freestyle, our design has a longer transmission distance and low packet loss rate. This is because we adopt lower transmission frequency and dual antenna. Lower transmission frequency has the advantage of less transmission loss. Dual antenna has the advantage of less packet loss.

\section{CONCLUSION}

This paper has designed and implemented a handheld ultrasonic video wireless transmission system. The interface of the wireless module has been selected. Through comparison, it is found that the wireless module with SDIO interface has the characteristics of low power consumption, high transmission speed, and stable connection. With the embedded Linux operating system, circuit design, software porting, our system has been fully implemented. Our system can be configured into two modes. In station mode, the data rate has been measured to be $367 \mathrm{Mbps}$ for UDP and 304Mbps for TCP. When the system is configured as AP mode, the data rate is $348 \mathrm{Mbps}$ for UDP and 298Mbps for TCP. The packet loss rates at different data rates and distances under UDP are tested. The test results show that the highest packet loss rate is $3.64 \%$ when the system is working in station mode, and $2.63 \%$ when working in AP mode. The transmission distance of the system can reach $9 \mathrm{~m}$. Our future work is to further improve the transmission power, so that our handheld system can have longer battery life time.

\section{ACKNOWLEDGMENTS}

This work was supported in part by National key R\&D program of China (Grant number 2016YFC0105002, 61674162, 2018YFB2100904, U1913601, 2018YFF01012500), Shenzhen Key Lab for RF Integrated Circuits, Shenzhen Shared Technology Service Center for Internet of Things, Shenzhen government funds (Grant numbers JCYJ20180305164616316).

\section{REFERENCES}

[1] R. D. Pozza, M. Loeff, (2010). Hand-Carried Ultrasound Devices in Pediatric Cardiology: Clinical Experience with Three Different Devices in 110 Patients, Journal of the American Society of Echocardiography, 1231-1237

[2] R. M. Khanwalla, K. T. (2017) Birkeland, Usefulness of Serial Measurements of Inferior Vena Cava Diameter By Vscan TM to Identify Heart Failure Patients at High Risk of Hospitalization, The American Journal of Cardiology, 1631-1636.

[3] Y. Lee, J. Kang, S. Yeo, (2014). A New Smart Probe System for a Tablet PC-based Point-of-Care Ultrasound Imaging System: Feasibility Study, 2014 IEEE International Ultrasonics Symposium Proceedings,1611-1614,.

[4] K. C. Kin, M. J. Kim, H. S. Joo, (2013). Smartphone-based Portable Ultrasound Imaging System : A Primary Result, 2013 Joint UFFC, EFTF and PFM Symposium, 2061-2063.

[5] T. Kei, A. Takashi, A. Takero, (2015). Usefulness of ACUSON Freestyle (Siemens), Wireless Ultrasound System, The Japanese journal of anesthesiology, 879-882.

[6] T. Zhu, (2013). Application and development of handheld ultrasound equipment in medical health field, Medical equipment in China,

[7] W. Zhou,Y. Zhang, L. Sun, J. Su, (2020). Application of embedded wireless video transmission system in Shipborne UAV, Software engineering, 1-4.

[8] W. Xie, Z. Wang, M. Ma, (2020). FEC algorithm for telemedicine data transmission, Medical and health equipment, 14-17. 